\title{
Early Hospital Outcome of Using Both Internal Mammary Arteries in Cairo University Hospital
}

Fouad M. Rasekh, Ahmed. S. Mahmoud*

Department of Cardiothoracic Surgery, Faculty of Medicine, Cairo University

*Corresponding authors: Ahmed Sayed Hussein, Mobile: (+20) 01090991111, E-mail: drahmed 755@gmail.com

\begin{abstract}
Background: Long-Using both mammary arteries in coronary artery bypass surgery has a good term results, but it is not recommended in old patients due to its technical difficulty and increased incidence of sternal wound complications. Several studies declared that bilateral internal mammary arteries (BIMA) grafting has a great benefit in patients aged 50-60 years, but this benefit does not extend to patients aged $>60$ years. This study was designed to analyze the early hospital results, and experience in preventing sternal wound complications for BIMA grafting in patients 50-60 years
\end{abstract} old.

Objective: To detect early outcome of using both internal mammary arteries in patients aged 50-60 years in Cairo University Hospital.

Patients and Methods: Clinical data and echocardiographic and coronary angiography data of 100 patients who underwent BIMA grafting for coronary artery disease between 2017 and 2019 were analyzed retrospectively to detect the early hospital results. 100 patients aged 50 to 60 years. The operation time, aortic clamp time, and cardiopulmonary bypass time were studied to analyze the operation difficulty. The flow and P.I were studied to analyze the coronary artery graft function. The left ventricular end-diastolic dimension (LVEDD) and left ventricular ejection fraction (LVEF) were studied to detect heart function.

Results: The operation time, aortic clamp time, and cardiopulmonary bypass time as well as the flow and pulsatility index were recorded. There was no incidence of sternal wound complications or graft occlusion. Furthermore, there was no significant difference in LVEF post-operation.

Conclusions: Using both mammary arteries in coronary artery bypass surgery is safe and effective for patients 5060 years old similar to younger patients $(<50$ years $)$.

Keywords: Coronary artery disease, Internal mammary arteries, Cairo University Hospital.

\section{INTRODUCTION}

Due to the increase in life expectancy, the proportion of coronary bypass surgery for elderly patients increases ${ }^{(\mathbf{1})}$. Due to the high rate of opening of the arterial ducts and its protective effect on the coronary course of patients ${ }^{(2)}$.

Several studies have shown that the use of both breast arteries in coronary artery bypass surgery is better than a single breast graft in protecting heart function and long-term follow-up after surgery ${ }^{(3,4)}$. However, due to the increased operation time, technical difficulties, and increased incidence of complications of sternal wounds, the application of BIMA vaccination is not widely used ${ }^{(5)}$. The use of the BIMA vaccination is about $6 \%$ worldwide and is only recommended for younger patients ${ }^{\left({ }^{6}\right)}$. Mohammadi et al ${ }^{(7)}$ reported that BIMA vaccination has great benefit in patients aged 5059 years.

Thus, this study was designed to analyze the experience of the operation, the graft function during surgery, early hospital outcomes, and the experience in preventing sternal complications of the use of both mammary arteries in coronary artery bypass surgery in patients 50-60 years old.

Aim of the present work was to detect early outcome of using both internal mammary arteries in patients aged 50-60 years in Cairo University Hospital.

\section{PATIENT AND METHODS}

From January 2017 to January 2019, 100 patients with coronary artery disease underwent BIMA grafting in our hospital.

Inclusion criteria: patients with multi vessel disease.

Exclusion criteria: 1) emergency surgery or patients with other critical diseases requiring concurrent surgery; 2) patients with severe cardiac failure or multiple organ dysfunction before surgery.

\section{Ethical and patients' approval:}

An approval of the study was obtained from Cairo University academic and ethical committee. Every patient signed an informed written consent for acceptance of the operation.

Operative methods

The surgeries were performed via median sternotomy. The BIMA and great saphenous vein were harvested. In 100 patients the internal mammary artery (IMA) was harvested using skeletonization technique (Fig. 1). After the IMA was harvested, heparin was given, and the distal end was cut off. Then, it was wrapped in warm wet gauze for preservation. All of the surgeries were performed using heart lung machine and warm antegrade cardioplegia. The distal and proximal end were anastomosed before unclamping of the aorta. The common grafting methods were as follows: the left 
internal mammary artery (LIMA) was anastomosed to the left anterior descending artery (LAD), the right internal mammary artery (RIMA) was anastomosed to the diagonal branch artery and obtuse marginal branch $(\mathrm{OM})$ and the great saphenous vein was anastomosed to the right coronary or its branches. All LIMAs were in situ grafts, while all RIMAs were free grafts (fig. 2).

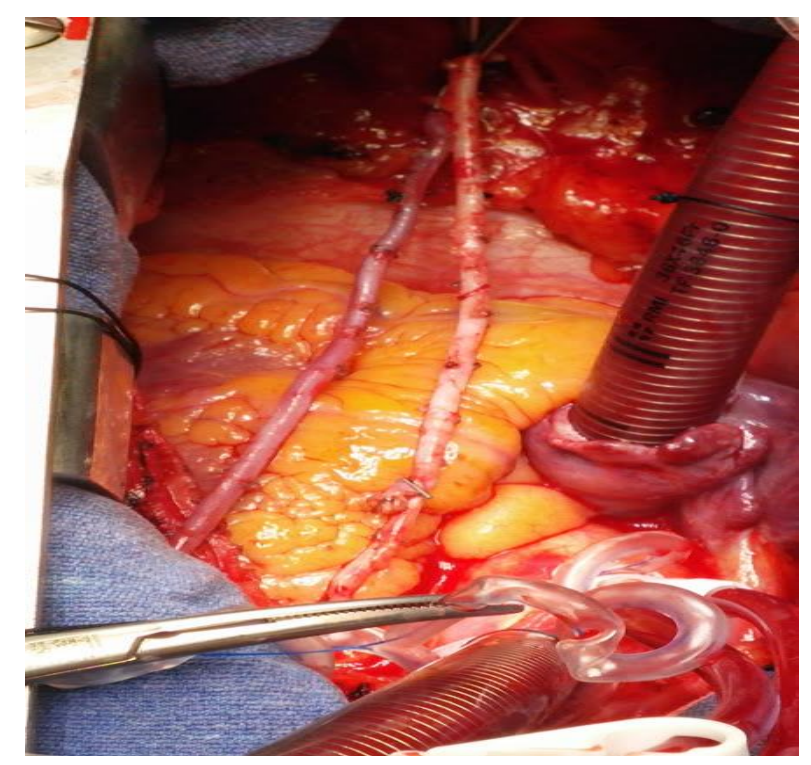

Figure (1): Skeletonized both internal mummeries.

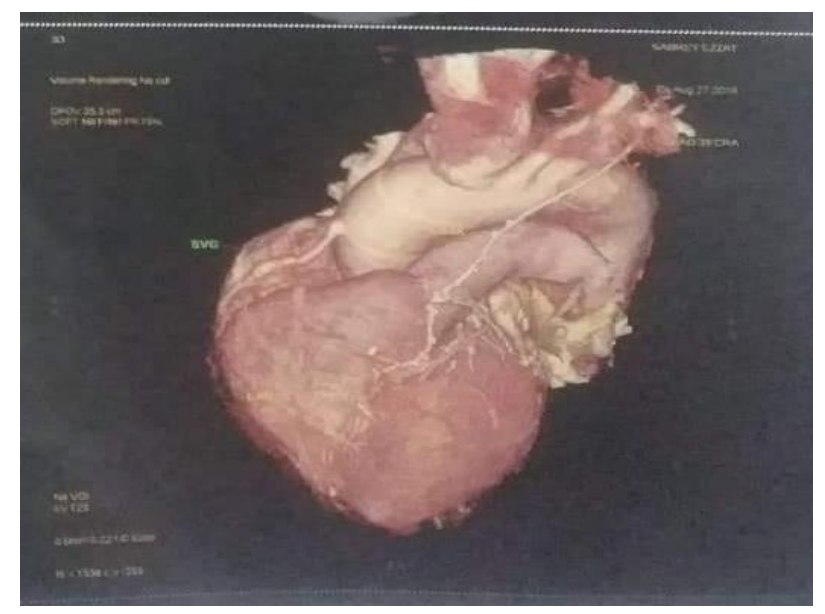

Figure (2): RIMA was free graft, and it was anastomosed to OM. RIMA, right internal mammary artery; OM, obtuse marginal branch.

Table (1): Operative details of arterial grafts

\begin{tabular}{|l|c|}
\hline LIMA-LAD & 100 \\
\hline RIMA-OM & 95 \\
\hline LIMA -D-LAD & 5 \\
\hline RIMA-D-OM & 5 \\
\hline
\end{tabular}

\section{Collection of the data:}

Preoperative data included the sex, age, diabetes, preoperative haemoglobin A1c, hypertension, body mass index (BMI), previous myocardial infraction, serum creatinine, hyperlipidaemia, cardiac function, and left ventricular ejection fraction (LVEF). Besides, the operative time, aortic cross clamp time, CPB time, number of distal coronary anastomosis, flowmetry of the LIMA, RIMA, and early postoperative complications were also recorded. The flowmetry of the IMA graft were measured by Veri Q system (Medistim, Sandakervn, Norway), and LVEF were recorded in every patient using echocardiography.

Mild sternal wound complications referred to sternal wound discharge without sternal rocking or wound infection. It could be cured by frequent dressing.

\section{Statistical analysis}

Data were analyzed using the software SPSS (Statistical Package for the Social Sciences) version 20. Quantitative variables were described using their means and standard deviations. The level of statistical significance was set at $5 \%(\mathrm{P} \leq 0.05)$. Highly significant difference was present if $\mathrm{P} \leq 0.001$.

\section{RESULTS}

Data of the study population: There were 100 patients with an average age of 50-60 years. The incidence males were $80 \%$ and females were $20 \%$. Rate of diabetes was $15 \%$, hypertension was $90 \%$, hyperlipidaemia was $80 \%$ and body mass index (BMI) was around $26 \%$. NO previous myocardial infraction, LVEF was normal preoperative, HbA1c was below 7.5 and serum creatinine was normal (Table 2).

Table (2): Characteristics of the Study Population

\begin{tabular}{|l|c|}
\hline AGE & 50-60 years \\
\hline MALES & $80 \%$ \\
\hline FEMALES & $20 \%$ \\
\hline $\begin{array}{l}\text { BMI (weight } \\
\text { kg/height in } \mathrm{m}^{2} \text { ) }\end{array}$ & Around $26 \%$ \\
\hline Diabetic & $15 \%$ \\
\hline hypertension & $90 \%$ \\
\hline hyperlipidemia & $80 \%$ \\
\hline HBA1c (\%) & Below 7.5 \\
\hline Cardiac function & normal \\
\hline
\end{tabular}

Operative data: The operative time, aortic clamp time and CPB time were recorded. The number of distal coronary anastomosis were around 3 to 4 . The flowmetry of LIMA was around $40-60 \mathrm{ml} / \mathrm{min}$ and below 2 , while they were $20-30 \mathrm{ml} / \mathrm{min}$ and below 2 for RIMA respectively. The intra-operative data of the study population are shown in Table (3).

Table (3): Operative data of the Study Population

\begin{tabular}{|l|c|}
\hline Operative time $(\mathrm{h})$ & $4.6 \pm 0.5$ \\
\hline Aortic clamp time $(\mathrm{min})$ & $60 \pm 15$ \\
\hline CBP $(\mathrm{min})$ & $75 \pm 20$ \\
\hline Number of distal coronary grafts & $3-4$ \\
\hline LIMA flow & $40-60 \mathrm{ml} / \mathrm{min}$ \\
\hline LIMA pulsatility index (PI) & Below 2 \\
\hline RIMA flow & $20-30 \mathrm{ml} / \mathrm{min}$ \\
\hline RIMA Pulsatility index (PI) & Below 2 \\
\hline
\end{tabular}


Post-operative Data: The operative mortality was zero. Two patients needed re-exploration for high drainage. Four patients showed elevated creatinine in early post-operative but did not require dialysis. Five patients developed atrial fibrillation that responded to medical cardioversion. NO patients needed prolonged ventilation. NO patients developed deep sternal wound infection only superficial wound discharge. Postoperative echocardiography was normal.

Table (4): Post-operative data

\begin{tabular}{|l|c|}
\hline Operative mortality & 0 \\
\hline Re-exploration & 2 \\
\hline RISING post-operative creatinine & 4 \\
\hline ATRIAL fibrillation & 5 \\
\hline Prolonged ventilation & 0 \\
\hline Deep sternal wound infection & 0 \\
\hline Post-operative echocardiography & Normal \\
\hline
\end{tabular}

\section{DISCUSSION}

The main findings of this study were: (1) Arterial grafting is more difficult than conventional coronary artery bypass grafting (CABG). The operation time, aortic clamp time, and $\mathrm{CPB}$ time were longer than conventional CABG, (2) The flowmetry of arterial grafts of left anterior descending branch (LIMA) and right internal mammary artery (RIMA) were good. (3) There was no incidence of common complications, such as graft occlusion and sternal wound complications.

In this study, BIMA were skeletonized and BIMA grafting was done using heart-lung machine. Since, the LIMA is used to graft the left coronary system, so the LIMA was anastomosed to the LAD and the diagonal branch artery if possible. The RIMA is used to revascularize the branches of circumflex artery and prevention of sternal complications.

The main factors which decrease the application of BIMA grafting are poor sternal wound healing and mediastinitis (especially for patients with BMI $>35 \mathrm{~kg} / \mathrm{m}^{2}$ ), elderly women, high HBA1c or patients with severe chronic obstructive respiratory dysfunction (COPD) ${ }^{(8)}$.

In this study, the incidence of deep sternal wound infection was $0 \%$. Although there were 20 diabetic patients in our study, our strategy to prevent sternal wound complications was as follows: 1) for diabetic patients, preoperative HbA1c should be controlled below $7.5 \%$. Several studies showed that elevated HBA1c was associated with high-risk factor of deep sternal wound infection ${ }^{(9)} .2$ ) The skeletonization of the IMA can cause little damage to the chest wall, and preserves the branches of the IMA and internal mammary vein. It is of great value to the healing of sternal wound and venous reflux ${ }^{(10)}$. So, in BIMA grafting, a number of guidelines have recommended skeletonization OF IMA to reduce the sternal wound infection ${ }^{(11,12)}$. 3) Sternal fixation was done by the "8shaped method". Four steel wires were used to fix the sternum.

\section{CONCLUSIONS}

Using both mammary arteries in coronary artery bypass surgery is safe and effective for patients (5060 years) similar to younger patients ( $<50$ years). BIMA grafting in patients (50-60 years) could also achieve a satisfactory early results.

\section{REFERENCES}

1. Itoh S, Kimura N, Adachi $\mathbf{H}$ et al. (2016): Is bilateral internal mammary arterial grafting beneficial for patients aged 75 or older? Circ J., 80: 1756-63.

2. Dimitrova K, Hoffman D, Geller C et al. (2012): Arterial grafts protect the native coronary vessels from atherosclerotic disease progression. Ann Thorac Surg., 94: 475-81.

3. Kelly R, Buth K, Légaré J (2012): Bilateral internal thoracic artery grafting is superior to other forms of multiple arterial grafting in providing survival benefit after coronary bypass surgery. J Thorac Cardiovasc Surg., 144: 1408-15.

4. Shi W, Hayward P, Tatoulis J et al. (2015): Are all forms of total arterial revascularization equal? A comparison of single versus bilateral internal thoracic artery grafting strategies. J Thorac Cardiovasc Surg., 150: 1526-33.

5. Kieser T, Lewin A, Graham M et al. (2011): Outcomes associated with bilateral internal thoracic artery grafting: the importance of age. Ann Thorac Surg., 92: 1269-75.

6. Itagaki S, Cavallaro P, Adams D et al. (2013): Bilateral internal mammary artery grafts, mortality and morbidity: an analysis of 1526360 coronary bypass operations. Heart, 99: 849-53.

7. Mohammadi S, Dagenais F, Doyle D et al. (2008): Age cut-off for the loss of benefit from bilateral internal thoracic artery grafting. Eur J Cardiothoracic Surg., 33: 977-82.

8. Pettinari M, Sergeant P, Meuris B (2015): Bilateral internal thoracic artery grafting increases long-term survival in elderly patients. Eur J Cardiothorac Surg., 47: 703-9.

9. Rollins K, Varadhan K, Dhatariya K et al. (2016): Systematic review of the impact of HbA1c on outcomes following surgery in patients with diabetes mellitus. Clin Nutr., 35: 308-16.

10. Kamiya H, Akhyari P, Martens A et al. (2008): Sternal microcirculation after skeletonized versus pedicled harvesting of the internal thoracic artery: a randomized study. J Thorac Cardiovasc Surg., 135: 32-7.

11. Gatti G, Maschietto $L$, Morosin M et al. (2017): Routine use of bilateral internal thoracic artery grafting in women: a risk factor analysis for poor outcomes. Cardiocasc Revasc Med., 18: 40-6.

12. Hashimoto M, Fukui T, Takanashi $S$ et al. (2015): Bilateral Internal Thoracic Artery Grafting: Is It Reasonable in Octogenarians? Ann Thorac Cardiovasc Surg., 21: 452-8. 